\title{
Characterization and Control of Conservative and Non-conservative Network Dynamics is
}

\author{
Matthias Wildemeersch ${ }^{\mathrm{a}, 1}$, Wai Hong Ronald Chan ${ }^{\mathrm{b}, 1}$, Elena Rovenskaya ${ }^{\mathrm{a}, \mathrm{c}}$, \\ Tony Q. S. Quek ${ }^{\mathrm{d}}$ \\ ${ }^{a}$ International Institute for Applied Systems Analysis (IIASA), Laxenburg, Austria \\ ${ }^{b}$ Stanford University, California, USA \\ ${ }^{c}$ Faculty of Computational Mathematics and Cybernetics, Lomonosov Moscow State \\ University, Moscow, Russia \\ ${ }^{d}$ Singapore University of Technology and Design (SUTD), Singapore
}

\begin{abstract}
Diffusion processes are instrumental to describe the movement of a continuous quantity in a network of interacting agents. Here, we present a framework for diffusion in networks and study in particular two classes of agent interactions depending on whether the total network quantity follows a conservation law. Focusing on probabilistic, asymmetric interactions between agents, we define how the dynamics of conservative and non-conservative networks relate to the weighted in-degree and out-degree Laplacians. For uncontrolled networks, we compare the convergence behavior of both types of networks as a function of the eigenvectors of the weighted graph Laplacians. For networks with exogenous controls, we also analyze convergence and provide a method to measure the difference between conservative and non-conservative network dynamics based on the comparison of their respective reachable sets. The presented network control framework enables the comparative study of the dynamic and asymptotic network behavior for conservative and non-conservative networks.
\end{abstract}

Keywords: multi-agent networks, diffusion process, directed graphs, graph Laplacian, network control, reachable set

\section{Introduction}

Multi-agent network dynamics received ample research interest over the last decade in the context of group coordination [2], distributed algorithms [3], net-

\footnotetext{
The material in this paper has been presented in part at the 2015 IEEE Conference on Decision and Control [1].

Email addresses: wildemee@iiasa.ac.at (Matthias Wildemeersch), whrchan@stanford.edu (Wai Hong Ronald Chan), rovenska@iiasa.ac.at 
work control [4], distributed optimization [5], consensus problems [6, 7, 8, 9, 10], and herding and flocking behavior [11]. Network dynamics involve inter-agent interactions that lead to the diffusion of a continuous quantity within a network $[12,13,14]$. In this work, we establish a probabilistic diffusion framework that describes in continuous time the movement of such a continuous quantity within a network, accounting for the stochasticity and the nature of the interactions between agents. Going beyond symmetric, unweighted graphs [15], we focus on weighted digraphs with asymmetric update rules. The main contribution of this framework is twofold. First, we make a connection between two linear update protocols and their corresponding network dynamics. Although these protocols are different in nature with regard to the conservation of total network quantity, they can result in identical network behavior under certain circumstances. Second, we enable the comparison between the protocols by studying the steady-state and transient behavior under both protocols, in the presence and absence of external control.

Many dynamical processes that occur over networks rely on pairwise interactions between network nodes that adjust their values according to a rule of interaction. There exists a large body of literature where the network dynamics are based on different graph Laplacian matrices [16]. A common feature of this literature is that the total amount of quantity present in the network does not follow a conservation law $[17,18,2,19,20]$. In this work instead, we present an inter-agent update rule that follows a conservation law and contrast it with a non-conservative update rule that is typically found in the literature. Considering both protocols, our framework can capture a wide range of network phenomena: financial and trade assets, biochemical systems, as well as human migration represent conservative flows [21,22], while the propagation of opinions follow non-conservative network dynamics. We derive the corresponding matrix differential equation that describes the diffusion of the considered quantity over the network, and highlight the differences in transient and stationary behavior for both update rules, taking into account the effects of network asymmetry.

Furthermore, we address network control by extending the homogeneous differential equation that describes the diffusion process to its inhomogeneous form. By doing so, we can model the addition of the considered quantity to the multi-agent network. We illustrate how constant control actions can result in changes of the system matrix that governs the network dynamics, and we define the convergence behavior of networks with exogenous excitation under given constraints on the input vector and network topology. When the control actions belong to a function space, we also provide a method to define the set of reachable network states based on the support function of non-empty closed convex sets. This method allows us to analyze how the conservative and non-conservative update protocol result in different reachable sets using the Hausdorff distance between these sets.

The remainder of the paper is structured as follows. Section 2 introduces the notation used throughout the paper and the Laplacian matrices that will be instrumental to characterize the network dynamics. Section 3 introduces two essential update rules to model diffusion in networks. Section 4 discusses the 
stability and convergence characteristics of conservative and non-conservative networks without control inputs, and Section 5 covers network control by extending the homogeneous equations to their inhomogeneous forms. Section 5 also presents a method to calculate the reachable set of network states. Finally, Section 6 provides some concluding remarks.

\section{Preliminaries}

We consider a population $\mathcal{V}$ of interacting agents $\mathcal{V}_{i}$, where $i \in \mathcal{I}=\{1,2, \ldots, n$ : $\left.n \in \mathbb{Z}^{+}\right\}$. All agents possess a continuous node property $S_{i}(t) \in \mathbb{R}, t \geq t_{0}$, and the node properties are gathered in the state vector $S(t)=\left[S_{1}(t) \ldots S_{n}(t)\right]^{\mathrm{T}}, S(t) \in$ $\mathbb{R}^{n}$. Assuming that $t_{0}=0$ and given the initial conditions $S(0)=S_{0}$, the node properties evolve over time according to a stochastic update process where property updates occur at times determined by a Poisson process. The probabilistic interactions between the agents can be described by a weighted digraph $G=(\mathcal{V}, \overrightarrow{\mathcal{E}}, w)$, where $\mathcal{V}$ is the set of agents and $\overrightarrow{\mathcal{E}}$ is the set of directed links $(i, j)$ between pairs of agents from $\mathcal{V}$. In this work, we consider $\mathcal{V}$ and $\overrightarrow{\mathcal{E}}$ to be constant over time. The weight function $w: \overrightarrow{\mathcal{E}} \mapsto \mathbb{R}^{+}$captures for each edge in the network the update rate as well as the liability between the interacting agents. The weighted adjacency matrix can be represented as

$$
A_{G}(i, j)= \begin{cases}w(i, j) & \text { if }(i, j) \in \overrightarrow{\mathcal{E}} \\ 0 & \text { otherwise }\end{cases}
$$

The weighted in-degree and out-degree matrices are diagonal matrices with diagonal elements given by

$$
\begin{aligned}
D_{G}^{(\text {in })}(j, j) & =\sum_{i} A_{G}(i, j), \\
D_{G}^{\text {(out) }}(i, i) & =\sum_{j} A_{G}(i, j) .
\end{aligned}
$$

Since the interactions between agents can be asymmetric, we define two Laplacians that refer to the in-degree and the out-degree of each node. We define the weighted in-degree and out-degree Laplacians as

$$
\begin{gathered}
L_{G}^{(\text {in })}=D_{G}^{(\text {in })}-A_{G}, \\
L_{G}^{\text {(out) }}=D_{G}^{\text {(out) }}-A_{G} .
\end{gathered}
$$

\section{Stochastic update rules}

Depending on the update rule applied in the probabilistic interactions between nodes, we characterize the flow dynamics of networks operating under different protocols. Here, we describe two main classes of linear update rules that result in linear, time-invariant matrix differential equations in the node 
property. These update rules are distinguished by the conservation or the nonconservation of the total property initially present in the network. The networks applying the conservative and non-conservative protocols will be referred to as conservative and non-conservative networks, respectively.

\subsection{Conservative networks}

We first consider a protocol where the total property in the network is conserved, i.e., $S_{t o t}=S_{1}(t)+\ldots+S_{n}(t)$ is constant over time. Conservative updating is relevant for the description of conservative flow dynamics between network nodes, including the flow of material and physical assets. In this respect, conservative networks are able to represent stylized instances of hydraulic networks, human mobility [21], or biochemical systems [22]. Here, agents obey the conservative update rule

$$
\begin{aligned}
& S_{i}(t+\Delta t)=S_{i}(t)+C_{i j} S_{j}(t) \\
& S_{j}(t+\Delta t)=\left(1-C_{i j}\right) S_{j}(t),
\end{aligned}
$$

where $i, j \in \mathcal{I},(i, j) \in \overrightarrow{\mathcal{E}}$. The parameter $C_{i j} \in(0,1]$ is a measure of liability or responsibility of agent $j$ towards agent $i$, and $\Delta t$ is an infinitesimal time interval. On every edge $(i, j) \in \overrightarrow{\mathcal{E}}$, a stochastic process takes place on the probability space $(\Omega, \mathcal{F}, \mathbb{P})$, with sample space $\Omega=\mathbb{R}_{+}$, the $\sigma$-algebra $\mathcal{F}$ of Borel sets on $\mathbb{R}_{+}$, and probability measure $\mathbb{P}$. We consider a counting process on the positive reals according to an independent, stationary Poisson process with rate $r_{i j}>0$. The counting process has also an interpretation as a ticking clock with exponentially distributed inter-event times. The protocol (P1) is executed for nodes $i$ and $j$ when the independent Poisson clock of $(i, j)$ ticks at time $t$. The following Lemma characterizes the property dynamics of the expected value of $S$ in conservative networks.

Lemma 1. Let $\bar{S}(t)$ denote the expected value of $S(t)$. The dynamics of the expected value for a network applying (P1) are defined by the governing equation

$$
\dot{\bar{S}}(t)=Q \bar{S}(t)
$$

where $Q=-L_{G}^{(\mathrm{in})}$, the weight function is defined as $w(i, j)=C_{i j} r_{i j}$, and

$$
Q_{i j}= \begin{cases}C_{i j} r_{i j} & \text { if } i \neq j \\ -\sum_{k \neq i} C_{k i} r_{k i} & \text { if } i=j\end{cases}
$$

Proof. We first note that the total update rate for a node $i \in \mathcal{V}$ is given by $r_{i}=\sum_{j} r_{i j}$, and that the total update rate of the network is given by $r=\sum_{i} r_{i}$. Assume that a global network clock is ticking at rate $r$. Then, the probability that the clock will activate edge $(i, j)$ is given by $r_{i j} / r$, where in the limit of large-scale networks $r \approx 1 / \Delta t$. Consequently, when an outgoing edge $(i, j)$ of node $i$ is triggered with probability $r_{i j} \Delta t$, the probabilistic update of the node 
properties involved in the update according to $(\mathrm{P} 1)$ is given by

$$
\begin{aligned}
& \bar{S}_{i}(t+\Delta t)-\bar{S}_{i}(t)=\sum_{j \neq i} r_{i j} \Delta t C_{i j} \bar{S}_{j}(t) \\
& \bar{S}_{j}(t+\Delta t)-\bar{S}_{j}(t)=-\sum_{j \neq i} r_{i j} \Delta t C_{i j} \bar{S}_{j}(t) .
\end{aligned}
$$

For a single node $i$, we account for the probability that property is added or subtracted, and therefore we can succinctly write

$$
\bar{S}_{i}(t+\Delta t)-\bar{S}_{i}(t)=\sum_{j \neq i} \Delta t\left(r_{i j} C_{i j} \bar{S}_{j}(t)-r_{j i} C_{j i} \bar{S}_{i}(t)\right) .
$$

Dividing by $\Delta t$ and taking the limit for $\Delta t \rightarrow 0$, we get a system of differential equations

$$
\dot{\bar{S}}(t)=-L_{G}^{(\mathrm{in})} \bar{S}(t)
$$

which concludes the proof.

Note that the assumption of large-scale networks can be dropped by considering that $r_{i j} \Delta t$ also represents the expected number of events of the Poisson process on edge $(i, j)$ in the interval $\Delta t$, which leads to (7) and (8). Considering (A), we notice that $\bar{S}(t)$ belongs to the class of continuously differentiable functions $\mathcal{C}^{1}[0, \infty)^{n}$. In the remainder of the paper, we refer to $Q$ as the system matrix. ${ }^{2}$

\subsection{Non-conservative networks}

We now consider networks where property diffuses between agents by means of a non-conservative update protocol, i.e., $S_{t o t}=S_{1}(t)+\ldots+S_{n}(t)$ can vary over time. This protocol is of interest for opinion dynamics [14, 3], or preference dynamics in cultural theory [23]. Here, agents obey a linear update rule according to the convex combination between the current property of node $i$ and the property of neighboring node $j[14]$

$$
S_{i}(t+\Delta t)=C_{i j} S_{j}(t)+\left(1-C_{i j}\right) S_{i}(t),
$$

whenever the Poisson clock of edge $(i, j) \in \overrightarrow{\mathcal{E}}$ ticks. The measure of confidence $C_{i j}$ takes values in the interval $(0,1]$. In other words, when the $(i, j)$-th Poisson clock activates the link between agents $i$ and $j$, agent $i$ is triggered to poll the property value of agent $j$ with a measure of confidence $C_{i j}$ and update its own value accordingly. The following Lemma characterizes the property dynamics in non-conservative networks.

\footnotetext{
${ }^{2}$ Alternatively, $Q$ can capture a deterministic equivalent model, where the parameters $r_{i j}$ enable us to weigh the interactions with each network node.
} 
Lemma 2. The dynamics of the expected value for a network applying (P2) are defined by the governing equation

$$
\dot{\bar{S}}(t)=Q \bar{S}(t),
$$

where $Q=-L_{G}^{(\text {out })}, w(i, j)=C_{i j} r_{i j}$, and

$$
Q_{i j}= \begin{cases}C_{i j} r_{i j} & \text { if } i \neq j, \\ -\sum_{k \neq i} C_{i k} r_{i k} & \text { if } i=j .\end{cases}
$$

Proof. With $r_{i j} \Delta t$ the probability that edge $(\mathrm{i}, \mathrm{j})$ is triggered, the probabilistic update of the property of node $\mathcal{V}_{i} \in \mathcal{V}$ under (P2) is given by

$$
\bar{S}_{i}(t+\Delta t)-\bar{S}_{i}(t)=\sum_{j \neq i}\left(r_{i j} \Delta t\right) C_{i j}\left(\bar{S}_{j}(t)-\bar{S}_{i}(t)\right) .
$$

Dividing by $\Delta t$ and taking the limit for $\Delta t \rightarrow 0$, we obtain the instance-averaged linear differential equations represented by (A) with $Q=-L_{G}^{\text {(out) }}$.

Note that Lemma 2 extends the basic consensus algorithm where $\dot{\bar{S}}_{i}(t)=$ $\sum_{j \in \mathcal{N}_{i}}\left(\bar{S}_{j}(t)-\bar{S}_{i}(t)\right)$, with $\mathcal{N}_{i}$ the neighborhood of $i$, to asymmetrically weighted updating. The relevance of the asymmetry will be further discussed in Section III.

Remark 1. Lemmas 1 and 2 explicitly link the protocols (P1) and (P2) to the system matrix. Consequently, the system matrix captures all relevant properties of diffusion over networks, i.e., the update protocol, the network topology, the inter-agent measures of liability/confidence, and the update rates. In the general case of weighted digraphs, the two protocols, although very different, result in system matrices $Q$ that differ only on the diagonal. In the following we will refer to weighted digraphs as asymmetric networks. When $Q$ is symmetric, the in-degree and out-degree Laplacians are identical, and the two protocols are therefore equivalent for symmetric matrices. For asymmetric matrices, (4) and (5) show that the conservative system matrix is the transpose of the nonconservative system matrix if the two corresponding digraphs have the same network topology with equal weights, but with all link directions reversed.

\section{Uncontrolled network dynamics}

In this section, we analyze the transient and steady-state characteristics of (A) based on the eigendecomposition of $Q$. Due to the construction of $Q$ as a Laplacian matrix, $Q$ always has the eigenvalue $q_{\mathrm{s}}=0$. Moreover, since all nondiagonal elements are non-negative, $Q$ is a Metzler matrix for which $\exp (Q t)$ is non-negative for $t \geq 0$. If $Q$ is diagonalizable ${ }^{3}$, then the solution to (A), the

\footnotetext{
${ }^{3}$ The system matrix $Q$ is diagonalizable if the algebraic and geometric multiplicities of the degenerate eigenvalues are identical. In case $Q$ is defective, the generalized eigenvector decomposition of $Q$ can be used, but this does not allow to decouple the system of differential equations.
} 
dynamic network behavior, can be written as

$$
\begin{aligned}
\bar{S}(t) & =\exp \left(A \Lambda A^{-1} t\right) S_{0} \\
& =A \operatorname{diag}\left(\exp \left(q_{k} t\right)\right) A^{-1} S_{0},
\end{aligned}
$$

where $A$ contains the unit right eigenvectors of $Q$ as columns, $A^{-1}$ contains the corresponding left eigenvectors of $Q$ as rows, $q_{k}$ represents the eigenvalues of $Q$, and $\Lambda=\operatorname{diag}\left(q_{k}\right)$. Also,

$$
\begin{aligned}
\bar{S}(t) & =\sum_{k} \exp \left(q_{k} t\right) v_{\mathrm{R}, k} v_{\mathrm{L}^{*}, k}^{\mathrm{T}} S_{0} \\
& =\sum_{k} c_{k} \exp \left(q_{k} t\right) v_{\mathrm{R}, k},
\end{aligned}
$$

where $v_{\mathrm{R}, k}$ and $v_{\mathrm{L}^{*}, k}$ are the unit right and corresponding left eigenvectors ${ }^{4}$ of $Q$ in column form, and $c_{k}=v_{\mathrm{L}^{*}, k}^{T} S_{0}$ are scalars. We notice from (14) that the eigenvalues and eigenvectors reflect the characteristic growth and decay rates of the system, as well as the dominant and weak nodes in the network. Concerning the eigenvalues of $Q$, Geršgorin's circle theorem states that all eigenvalues of $Q$ reside in the complex plane within the union of the disks $D_{i}=\{z \in \mathbb{C}: \mid z-$ $\left.Q_{i i}\left|\leq \sum_{j \neq i}\right| Q_{i j} \mid\right\}, i \in \mathcal{I}$. According to this theorem, $Q$ matrices constructed in compliance with (P1) or (P2) have nonpositive eigenvalues including zero, such that the node quantities are stable and converge asymptotically to a steady state. The stationary network state is given by

$$
\lim _{t \rightarrow \infty} \bar{S}(t)=c_{\mathrm{s}} v_{\mathrm{R}, \mathrm{s}}
$$

where $c_{\mathrm{S}}=v_{\mathrm{L}^{*}, \mathrm{~s}}^{T} S_{0}$ and $v_{\mathrm{R}, \mathrm{s}}$ are the scalar and unit right eigenvector corresponding to $q_{\mathrm{s}}=0$. We now illustrate the dynamics for different strongly connected network classes using the network presented in Fig. 1.

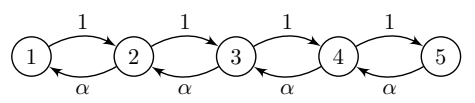

Figure 1: Graph $P_{5}$ with asymmetric link weights used to illustrate the transient and steadystate behavior of the two protocols.

\subsection{Conservative asymmetric networks}

For conservative networks, we formulate the following Lemma for the stationary behavior.

Lemma 3. The stationary value of a strongly connected asymmetric network with diagonalizable system matrix applying $(\mathrm{P} 1)$ is given by $v_{\mathrm{R}, \mathrm{s}}$ scaled by $c_{\mathrm{s}}=$ $\sum_{i} S_{0, i} / \Psi$ where $\Psi$ is the sum of the entries of $v_{\mathrm{R}, \mathrm{s}}$.

\footnotetext{
${ }^{4}$ The left eigenvectors $v_{\mathrm{L}^{*}, k}$ are only unit in the case where $Q$ is symmetric.
} 


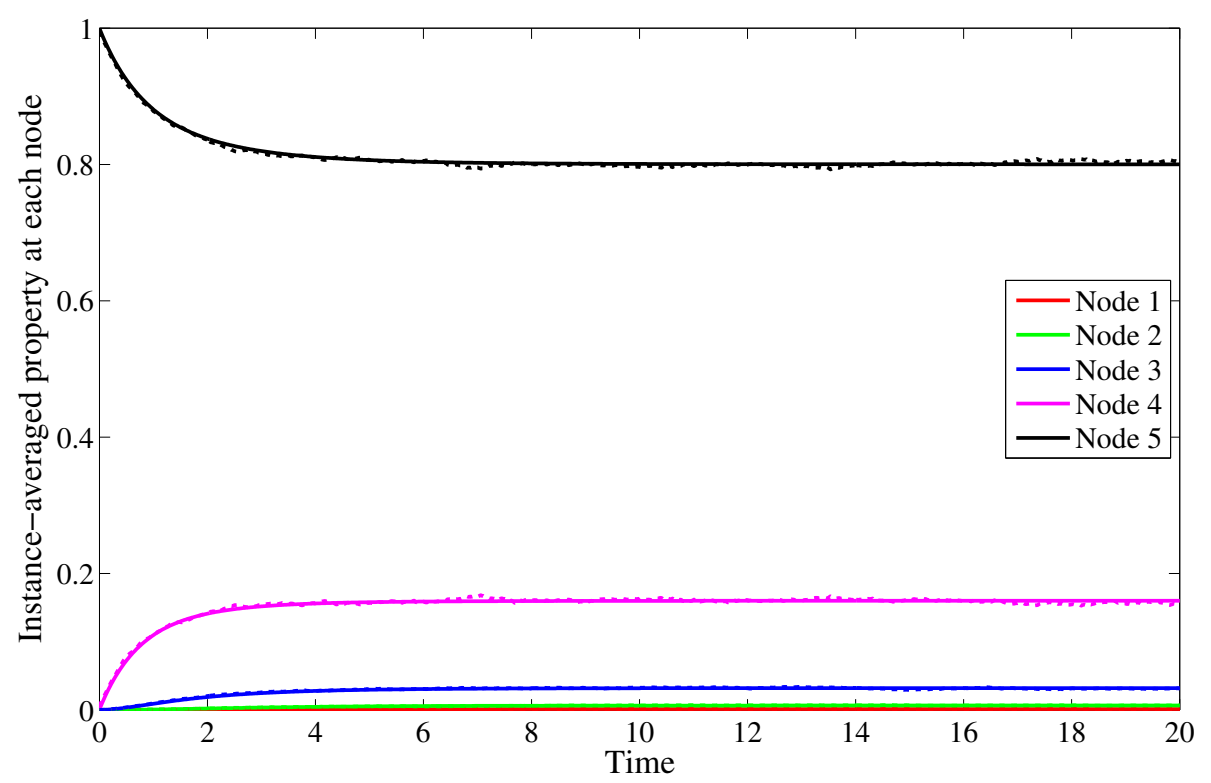

Figure 2: Comparison of instance-averaged node property obtained through Monte Carlo simulations (dotted lines) and expected property obtained by eigendecomposition of the matrix $Q$ (solid lines) for the asymmetric network in Fig. 1 with $\alpha=0.2$ under (P1). For the simulations, 5000 trials were performed, and each timestep was discretized into 1000 subunits. In both cases, the initial conditions $S_{0}=\left[\begin{array}{lllll}0 & 0 & 0 & 0 & 1\end{array}\right]$ were adopted.

Proof. The column-sum of $Q=-L_{G}^{(\text {in) }}$ is zero, and $Q$ thus has a steady-state left eigenvector $v_{\mathrm{L}^{*}, \mathrm{~s}}$ with equal components. In view of the construction of $Q=-L_{G}^{(\mathrm{in})}$, we find that $c_{\mathrm{s}}=v_{\mathrm{L}^{*}, \mathrm{~s}}^{T} S_{0}=\sum_{i} S_{0, i} / \Psi$, where the scaling is due to the right unit eigenvector. Considering that $\lim _{t \rightarrow \infty} \bar{S}(t)=c_{\mathrm{s}} v_{\mathrm{R}, \mathrm{s}}$, the proof is concluded.

In an asymmetric network, the stationary node values are imbalanced due to the unequal components in $v_{\mathrm{R}, \mathrm{s}}$. When asymmetric liabilities between nodes occur, the stationary distribution will favor attractive over repulsive nodes, and uniform spreading does not occur as a consequence of the conservation of total property. We illustrate this finding for the network depicted in Fig. 1 with $\alpha=0.2$, and we present in Fig. 2 the evolution of $\bar{S}_{i}(t)$ over time. In the latter plot, we generate $\bar{S}_{i}(t)$ by empirically generating sample paths based on the update rule (P1), and analytically determining the expected property from (14).

\subsection{Non-conservative asymmetric networks}

For networks that apply the non-conservative update rule (P2), we formulate the following Lemma for the stationary behavior. 
Lemma 4. A strongly connected asymmetric network with diagonalizable system matrix following the non-conservative update rule (P2) always achieves consensus. The consensus value $c_{\mathrm{v}}=c_{\mathrm{s}} / \sqrt{n}$ in the infinite time horizon is the following weighted average of $S_{0}$

$$
c_{\mathrm{v}}=\frac{1}{\sqrt{n}} v_{\mathrm{L}^{*}, \mathrm{~s}}^{\mathrm{T}} S_{0} .
$$

Proof. Since the row-sum of $Q=-L_{G}^{(\text {out })}$ is zero, $v_{\mathrm{R}, \mathrm{s}}$ has equal components. Hence, strongly connected networks with asymmetric updating always achieve agreement. Considering $\lim _{t \rightarrow \infty} \bar{S}(t)=c_{\mathrm{s}} v_{\mathrm{R}, \mathrm{s}}$ with $c_{\mathrm{s}}=v_{\mathrm{L}^{*}, \mathrm{~s}}^{T} S_{0}$, the consensus value can be found by determining the row of $A^{-1}$ corresponding to $q_{\mathrm{s}}=0$. For an asymmetric matrix, $v_{\mathrm{L}^{*}, \mathrm{~s}}$ can be calculated based on the initial conditions $P(0)=I_{n}=A^{-1} A$ and the knowledge of the unit eigenvector $v_{\mathrm{R}, \mathrm{s}}$. The result is then scaled by the magnitude of the entries of $v_{\mathrm{R}, \mathrm{s}}$ to obtain $c_{\mathrm{v}}=$ $\frac{1}{\sqrt{n}} v_{\mathrm{L}^{*}, \mathrm{~s}}^{\mathrm{T}} S_{0}$.

We illustrate in Fig. 3 the consensus behavior for the network depicted in Fig. 1 with $\alpha=0.2$. Here, $c_{\mathrm{v}}$ is heavily weighted towards node 5 , whose inward link weight exceeds its outward link weight. This means that node 5 is heavily polled by its neighbors. Such a trend is reminiscent of the availability heuristic, where subjects that are encountered or recalled more often are given more thought and emphasis [24].

\subsection{Symmetric networks}

In a strongly connected symmetric network, the steady-state right eigenvector has components of equal magnitude in all dimensions regardless of the update rule. We can interpret this as an equal sharing of resources in conservative networks, and as consensus among all agents at a value corresponding to the average of the initial conditions at all the nodes in non-conservative networks $[19,17,20,14]$. We illustrate this conclusion by observing $\bar{S}_{i}(t)$ over time in Fig. 4 for the network depicted in Fig. 1 with $\alpha=1$. In Fig. 4, we generate $\bar{S}_{i}(t)$ by both empirically generating sample paths based on the update rules (P1) and (P2), which are equivalent in this case, and analytically determining the expected property from (14).

\section{Network control by exogenous excitation}

To model the exogenous addition and subtraction of property to multi-agent networks, we extend the homogeneous equation (A) to include an inhomogeneous term

$$
\dot{\bar{S}}(t)=Q \bar{S}(t)+U(t) .
$$

For all $t$, the input vector $U(t)$ belongs for each $t \geq 0$ to $\mathcal{U}$, where $\mathcal{U}$ is a convex set in $\mathbb{R}^{n}$ and $U(\cdot) \in \hat{\mathcal{C}}[0, \infty)^{n}$ with $\hat{\mathcal{C}}$ the class of piecewise continuous functions. 


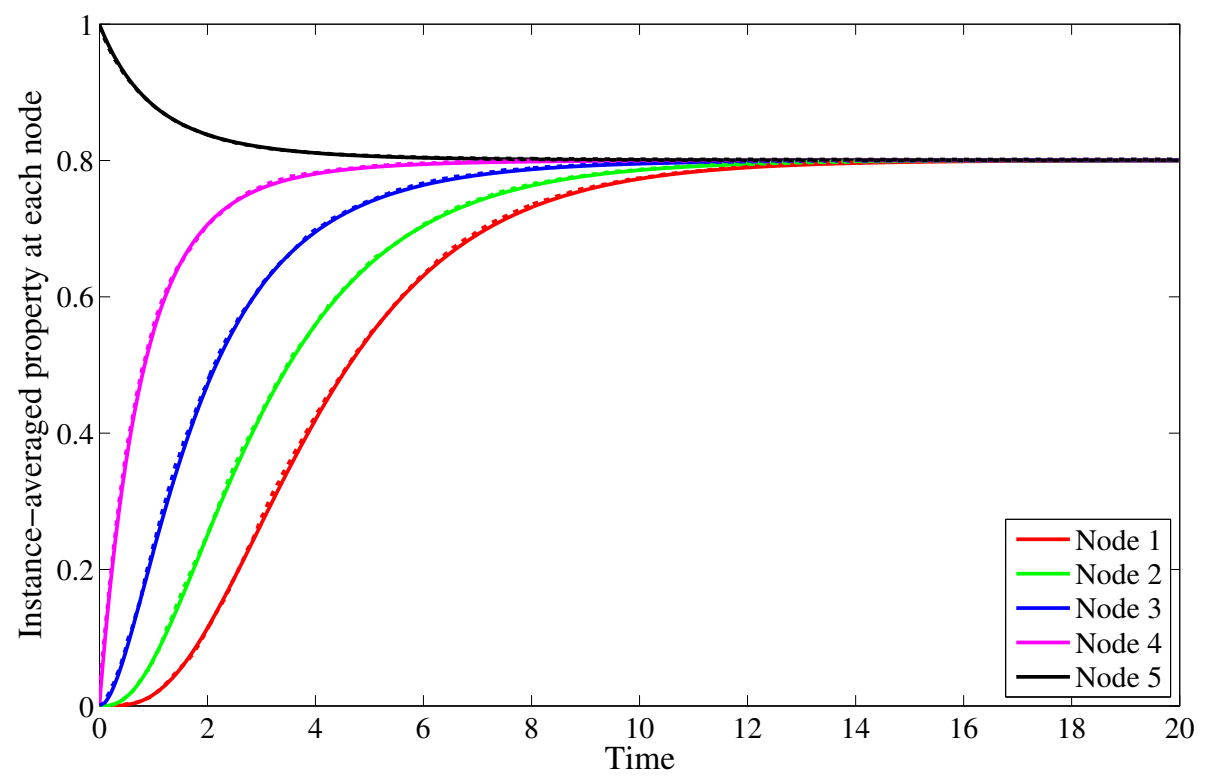

Figure 3: Comparison of instance-averaged property obtained numerically (dotted lines) and analytically (solid lines) for the asymmetric network in Fig. 1 with $\alpha=0.2$ under (P2). Simulation parameters are identical to those in Fig. 2.

For time-invariant matrix $Q$, the solution to (B), the dynamic network behavior with external control, is the Carathéodory solution

$$
\bar{S}(t)=\bar{S}_{\mathrm{h}}(t)+\int_{0}^{t} \exp (Q(t-\tau)) U(\tau) d \tau,
$$

where $\bar{S}_{\mathrm{h}}(t)=\exp (Q t) S_{0}$. This can be interpreted as the sum of the solution to the homogeneous equation (A) and the convolution of the input $U(t)$ with the impulse response to (B) for every node.

Definition 1. The linear system (B) is said to be positive if and only if for every non-negative initial state $S_{0}$ and every non-negative input $U(t), \bar{S}(t)$ is non-negative [25].

By construction, the system matrix $Q$ is a Metzler matrix, i.e., $Q_{i j} \geq 0, i \neq j$, which is a necessary and sufficient condition for the positivity of the system [25]. Note that $\mathbb{R}_{+}^{n}$ is a positive invariant set for the system defined by (A), which indicates that for each non-negative $S_{0}$ the trajectory remains in $\mathbb{R}_{+}^{n}$, both for conservative and non-conservative networks. The positivity of the network properties is also guaranteed for the linear system represented in (B) as long as the inputs are non-negative, and (B) represents a positive linear system. Positive systems often arise in realistic applications, such as in transport networks, storage systems, and stochastic models where the state variables and probabilities take non-negative values. 


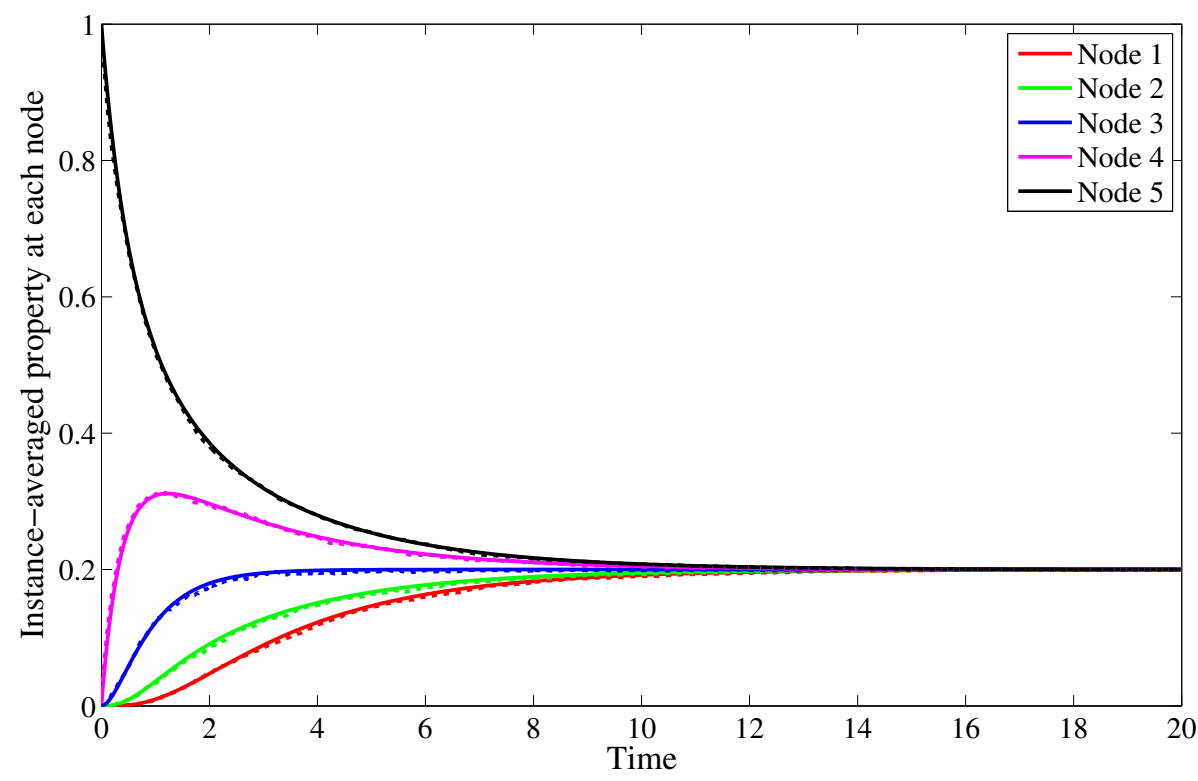

Figure 4: Comparison of instance-averaged property obtained numerically (dotted lines) and analytically (solid lines) for the symmetric network in Fig. 1 with $\alpha=1$. The simulation parameters are identical to those in Fig. 2.

In the following subsections, we first study the convergence of conservative and non-conservative network dynamics in the presence of exogenous inputs. We also present a methodology based on the support function to characterize the reachable sets at a given time $t$ for networks with exogenous control. This methodology allows us to compare the reachable sets of conservative and nonconservative networks in terms of the Hausdorff distance.

\subsection{Asymptotic behavior of controlled network dynamics}

In this section, we analyze the convergence behavior of the inhomogeneous equation (B) both for singular and non-singular system matrices. Although the system matrices used in (A) are by construction singular negative semidefinite matrices, we will illustrate how network control can result in modifications of $Q$ and in some cases in a non-singular $Q$-matrix. The solution to the inhomogeneous equation is given in (17) for an input vector $U(t) \in \mathcal{U}, \forall t \geq 0$. It is relevant to study the special case of constant input vectors, for instance when the input vectors belong to the boundary of admissible set $\partial \mathcal{U}$. For constant 
input vectors and diagonalizable $Q$, the solution to (B) is given by

$$
\begin{aligned}
& \bar{S}(t)=A \exp (t \Lambda) A^{-1} S_{0}+\int_{0}^{t} A \exp ((t-\tau) \Lambda) A^{-1} U \mathrm{~d} \tau \\
& =\sum_{k} c_{k} \exp \left(q_{k} t\right) v_{\mathrm{R}, k}+\sum_{k} \int_{0}^{t} \exp \left(q_{k}(t-\tau)\right) v_{\mathrm{R}, k} v_{\mathrm{L}^{*}, k}^{T} U \mathrm{~d} \tau \\
& =\sum_{k} c_{k} \exp \left(q_{k} t\right) v_{\mathrm{R}, k}+\sum_{k} u_{k} \int_{t}^{0} \exp \left(q_{k}(t-\tau)\right) \mathrm{d}(t-\tau) v_{\mathrm{R}, k},
\end{aligned}
$$

where $u_{k}=v_{\mathrm{L}^{*}, k}^{T} U$. This result can be further developed for singular and nonsingular system matrix.

Singular $Q$ : When $Q$ is singular with single zero eigenvalue, we get

$$
\begin{aligned}
\bar{S}(t)= & \sum_{k} c_{k} \exp \left(q_{k} t\right) v_{\mathrm{R}, k} \\
& +\sum_{k \neq 1} u_{k} q_{k}^{-1}\left(\exp \left(q_{k} t\right)-1\right) v_{\mathrm{R}, k}+u_{\mathrm{s}} t_{\mathrm{R}, \mathrm{s}},
\end{aligned}
$$

with $u_{\mathrm{s}}$ and $v_{\mathrm{R}, \mathrm{s}}$ the scalar and eigenvector corresponding to $q_{\mathrm{s}}=0$. By taking the limit over time, we get

$$
\lim _{t \rightarrow \infty} \bar{S}(t)=c_{\mathrm{s}} v_{\mathrm{R}, \mathrm{s}}-\sum_{k \neq 1} u_{k} q_{k}^{-1} v_{\mathrm{R}, k}+\lim _{t \rightarrow \infty} u_{\mathrm{s}} t v_{\mathrm{R}, \mathrm{s}} .
$$

Since $u_{\mathrm{s}}$ only equals zero for $U=0,(20)$ illustrates that the stationary network state under constant input $U$ is unbounded both for conservative and nonconservative networks.

Non-singular $Q$ : As $Q$ has no zero eigenvalues, (18) can be written as

$$
\bar{S}(t)=\sum_{k} c_{k} \exp \left(q_{k} t\right) v_{\mathrm{R}, k}+\sum_{k} u_{k} q_{k}^{-1}\left(\exp \left(q_{k} t\right)-1\right) v_{\mathrm{R}, k},
$$

and the steady-state behavior is given by

$$
\lim _{t \rightarrow \infty} \bar{S}(t)=S^{*}=c_{\mathrm{s}} v_{\mathrm{R}, \mathrm{s}}-\sum_{k} u_{k} q_{k}^{-1} v_{\mathrm{R}, k} .
$$

Alternatively, the steady state can also be found by means of the inverse of $Q$ as follows

$$
S^{*}=-Q^{-1} U \text {. }
$$

Both formulations allow us to define the set of reachable stationary network states $\mathcal{S}(\infty) \subset \mathbb{R}^{n}$ as a function of the set of admissible control values $\mathcal{U}$.

Remark 2. In Section 4, we demonstrated that the stationary behavior of uncontrolled networks only depends on the left and right eigenvector of $Q$ corresponding to $q_{\mathrm{s}}=0$. As opposed to these results, the stationary behavior of 
networks under control is a function of all eigenvectors of $Q$, which can be observed in (20) and (22). Therefore, while the uncontrolled non-conservative dynamics always led to consensus, this is not the case anymore for controlled non-conservative networks. The set of reachable network states in steady state for uncontrolled non-conservative networks is represented by a point in $\mathbb{R}_{+}^{n}$, while the set of reachable network states for controlled non-conservative networks does not have this restriction. In fact, we will observe that the presence of stubborn agents in the network results in polarization of the opinions in steady state.

We present now an example of controlled network dynamics where the system matrix is modified and in general non-singular. We consider the scenario of opinion dynamics where a set of agents has constant opinion. Adopting the definition proposed in [14], as well as the non-conservative update rule (P2), an agent is called stubborn if it does not adjust its own value based on the values of neighboring nodes. In these networks, the out-degree of a stubborn agent is zero and the corresponding row of $Q$ is a zero-row. For example, in the presence of two stubborn agents $a_{1}=2$ and $a_{2}=n$, the governing equation can be written as

$$
\left[\begin{array}{c}
\dot{\bar{S}}_{1} \\
\vdots \\
\dot{\bar{S}}_{n}
\end{array}\right]=\left[\begin{array}{cccc}
Q_{1,1} & Q_{1,2} & \cdots & Q_{1, n} \\
0 & 0 & \cdots & 0 \\
Q_{3,1} & Q_{3,2} & \cdots & Q_{3, n} \\
\vdots & \vdots & \ddots & \vdots \\
0 & 0 & \cdots & 0
\end{array}\right]\left[\begin{array}{c}
\bar{S}_{1} \\
\vdots \\
\bar{S}_{n}
\end{array}\right]
$$

which can be reformulated as

$$
\begin{array}{r}
{\left[\begin{array}{c}
\dot{\bar{S}}_{1}^{\prime} \\
\vdots \\
\dot{\bar{S}}_{n-2}^{\prime}
\end{array}\right]=\left[\begin{array}{cccc}
Q_{1,1}^{\prime} & Q_{1,2}^{\prime} & \cdots & Q_{1, n-2}^{\prime} \\
\vdots & \vdots & \ddots & \vdots \\
Q_{n-2,1}^{\prime} & Q_{n-2,2}^{\prime} & \cdots & Q_{n-2, n-2}^{\prime}
\end{array}\right]\left[\begin{array}{c}
\bar{S}_{1}^{\prime} \\
\vdots \\
\bar{S}_{n-2}^{\prime}
\end{array}\right]} \\
+\left[\begin{array}{cc}
\vdots & \vdots \\
B_{k, a_{1}} & B_{k, a_{2}} \\
\vdots & \vdots
\end{array}\right]\left[\begin{array}{c}
\bar{S}_{a_{1}} \\
\bar{S}_{a_{2}}
\end{array}\right],
\end{array}
$$

where $B_{k, a_{1}}$ and $B_{k, a_{2}}$ are the $a_{1}$-th and $a_{2}$-th columns of $Q$ with the $a_{1}$-th and $a_{2}$-th entries omitted. This gives us

$$
\dot{\bar{S}}^{\prime}(t)=Q^{\prime} \bar{S}^{\prime}(t)+B U_{\text {st }},
$$

where $\bar{S}^{\prime}$ and $Q^{\prime}$ represent the reduced state space and reduced system matrix, and $U_{\text {st }}=\left[\bar{S}_{a_{1}} \cdots \bar{S}_{a_{n}}\right]^{T}$ contains the static quantities of the stubborn agents. Stubborn agents allow for a reduction of the state space and a formulation equivalent to (B) with constant but possibly different inputs for different stubborn nodes and $U=B U_{\mathrm{st}}$. The reduced state space excludes the stubborn agents and the reduced system matrix excludes the rows and columns corresponding to these agents. The system matrix $Q^{\prime}$ is still a Metzler matrix, but 
without the control term in (26), $Q^{\prime}$ does not reflect the dynamics captured by (P2) in the strict sense. Without stubborn agents, $Q$ has by construction eigenvalue $q_{\mathrm{s}}=0$ and is singular, in which case we observe from (20) that BIBO (bounded-input, bounded-output) stability is not guaranteed and the system is only marginally stable. In the presence of stubborn agents and with constant $U$, it is known that bounded-input bounded-output (BIBO) stability of (26) is guaranteed iff all eigenvalues of $Q^{\prime}$ have negative real components. Let $\mathcal{I}_{\text {st }}=\left\{i \mid \bar{S}_{i}(t)\right.$ is constant $\}$ be the index set of stubborn agents, then we can write $Q_{i i}=-\sum_{j \neq i, j \notin \mathcal{I}_{\mathrm{st}}} Q_{i j}-\sum_{j \neq i, j \in \mathcal{I}_{\mathrm{st}}} Q_{i j}$, and the row sum of $Q^{\prime}$ satisfies

$$
Q_{i i}^{\prime}+\sum_{j \neq i} Q_{i j}^{\prime} \leq 0
$$

Thus, $Q^{\prime}$ does not have a zero eigenvalue in general and is non-singular in general. If each agent is connected to at least one stubborn agent, $Q^{\prime}$ is strictly diagonally dominant and we can write

$$
Q_{i i}^{\prime}+\sum_{j \neq i} Q_{i j}^{\prime}<0
$$

According to Geršgorin's circle theorem, all eigenvalues of $Q^{\prime}$ reside in the complex plane within the union of the disks $D_{i}=\left\{z \in \mathbb{C}:\left|z-Q_{i i}^{\prime}\right| \leq\right.$ $\left.\sum_{j \neq i}\left|Q_{i j}^{\prime}\right|\right\}, 1 \leq i \leq n^{\prime}$ with $n^{\prime}$ the dimension of $Q^{\prime}$. As $\left|Q_{i i}^{\prime}\right|>\sum_{j \neq i}\left|Q_{i j}^{\prime}\right|$ for a strictly diagonally dominant matrix, $\mathcal{Q}^{\prime}$ cannot have a zero eigenvalue and is invertible. Under these conditions, the network state converges to $\bar{S}^{\prime *}=$ $-Q^{\prime-1} B U_{\text {st }}$, as described in (22) and (23).

\subsection{Reachable set: analysis and comparison}

It is very instructive to study the reachable set at a given time $t$, which is the set of states that can be reached by using all admissible controls. As opposed to Section 5.1 where we studied the asymptotic network behavior under constant controls, we consider in this section a class of possibly time-varying controls that belong to a function space. The reachable set for conservative and nonconservative networks can be expressed as

$$
\begin{aligned}
\mathcal{S}(t) \equiv & \mathcal{S}\left(0, t, \mathcal{M}_{0}\right)=\left\{S(t) \in \mathbb{R}^{n} \mid S(t)=e^{t Q} S_{0}\right. \\
& \left.+\int_{0}^{t} e^{(t-\tau) Q} U(\tau) \mathrm{d} \tau, S_{0} \in \mathcal{M}_{0}, U(\tau) \in \mathcal{U}\right\},
\end{aligned}
$$

where $\mathcal{M}_{0}$ is the set of possible initial values $S_{0}$ and $\mathcal{U}$ is the compact set of admissible control values in $\mathbb{R}^{n} .{ }^{5}$ In view of conservative and non-conservative networks, we aim to describe the differences between the corresponding reachable sets at a given time $t$ when the dynamics take place over the same network.

\footnotetext{
${ }^{5}$ Note that for linear systems the reachable sets for open and closed-loop control are the same.
} 
To avoid confusion, we will indicate the system matrices for the conservative and non-conservative network dynamics by $Q_{\mathrm{c}}$ and $Q_{\mathrm{nc}}$ respectively. When the network structure is the same, $Q_{\mathrm{c}}$ differs from $Q_{\mathrm{nc}}$ only on the diagonal. Different methods exist to describe the reachable set, for instance by using the maximal principle [26] or by means of ellipsoidal methods that allow to numerically calculate approximations of the reachable set in terms of inner and outer bounds [27]. Here, for the characterization of reachable sets we propose to make use of the support function, which is widely applied in the analysis of convex sets [28]. The reachable set can be written also by means of the Minkowski sum

$$
\mathcal{S}(t)=e^{t Q} \mathcal{M}_{0}+\int_{0}^{t} e^{(t-\tau) Q} \mathcal{U} \mathrm{d} \tau
$$

The Minkowski sum and the linear transformations in (30) preserve compactness and convexity [28]. Therefore, if the initial set $\mathcal{M}_{0}$ and set of admissible controls $\mathcal{U}$ are compact and convex, then the reachable set $\mathcal{S}(t)$ is also compact and convex. Every non-empty compact convex set $\mathcal{F}$ is uniquely determined by its support function $c(\mathcal{F}, \psi)$, which is defined as

$$
c(\mathcal{F}, \psi)=\sup _{f \in \mathcal{F}}\langle f, \psi\rangle
$$

where $\langle.,$.$\rangle represents the inner product on \mathbb{R}^{n}$. For $\psi \in \mathcal{S}_{n}(0,1)=\{x \mid\|x\|=$ $1\}$ and $\|\cdot\|$ the $\ell^{2}$-norm, the support function represents the signed distance between the origin and the hyperplane $\Gamma_{\psi}=\{x \mid\langle x, \psi\rangle=c(\mathcal{F}, \psi)\}$. If $c\left(\mathcal{F}_{1}, \psi\right)=$ $c\left(\mathcal{F}_{2}, \psi\right), \forall \psi \in \mathbb{R}^{n}$, then $\mathcal{F}_{1}=\mathcal{F}_{2}$. Since $c(\mathcal{F}, k \psi)=k \cdot c(\mathcal{F}, \psi), \forall k \geq 0$, the support function can be used with a restriction of $\psi$ to the unit sphere $\mathcal{S}_{n}(0,1)$. We propose to use the Hausdorff metric to measure the distance between the reachable sets of the conservative and non-conservative networks at a given time $t$. The Hausdorff distance is a metric that describes the distance between subsets in a metric space and is defined as

$$
h\left(\mathcal{F}_{1}, \mathcal{F}_{2}\right)=\min _{r \geq 0}\left\{r \mid \mathcal{F}_{1} \subset \mathcal{F}_{2}+\mathcal{B}_{n}(0, r), \mathcal{F}_{2} \subset \mathcal{F}_{1}+\mathcal{B}_{n}(0, r)\right\}
$$

where the $n$-dimensional ball around the origin with radius $r$ is represented by $\mathcal{B}_{n}(0, r)=\{x \mid\|x\| \leq r\}$. In the following lemma, we present an upper bound for the Hausdorff distance between the reachable sets of conservative and nonconservative networks.

Theorem 1. The Hausdorff distance between the reachable sets of conservative and non-conservative networks, indicated by $\mathcal{S}_{\mathrm{c}}$ and $\mathcal{S}_{\mathrm{nc}}$ respectively, can be upper bounded as

$$
\begin{aligned}
& h\left(\mathcal{S}_{\mathrm{c}}(t), \mathcal{S}_{\mathrm{nc}}(t)\right) \\
& \leq \sup _{m \in \mathcal{M}_{0}}\|m\|\|A-B\|_{\mathrm{F}}+\sup _{u \in \mathcal{U}}\|u\|\|C-D\|_{\mathrm{F}},
\end{aligned}
$$

with $\|\cdot\|_{\mathrm{F}}$ the Frobenius norm, and where $A^{\mathrm{T}}=e^{t Q_{\mathrm{c}}}, B^{\mathrm{T}}=e^{t Q_{\mathrm{nc}}}$,

$$
C^{\mathrm{T}}= \begin{cases}Q_{\mathrm{c}}^{-1}\left(e^{t Q_{\mathrm{c}}}-I_{n}\right) & \text { for } Q_{\mathrm{c}} \text { nonsingular }, \\ t \sum_{k=0}^{\infty} \frac{1}{k+1} \frac{t^{k} Q_{\mathrm{c}}^{k}}{k !} & \text { for } Q_{\mathrm{c}} \text { singular }\end{cases}
$$


and

$$
D^{\mathrm{T}}= \begin{cases}Q_{\mathrm{nc}}^{-1}\left(e^{t Q_{\mathrm{nc}}}-I_{n}\right) & \text { for } Q_{\mathrm{nc}} \text { nonsingular }, \\ t \sum_{k=0}^{\infty} \frac{1}{k+1} \frac{t^{k} Q_{\mathrm{nc}}^{k}}{k !} & \text { for } Q_{\mathrm{nc}} \text { singular } .\end{cases}
$$

Proof. The support functions for conservative and non-conservative reachable sets are defined as

$$
\begin{gathered}
c\left(\mathcal{S}_{\mathrm{c}}(t), \psi\right)=c\left(e^{t Q_{\mathrm{c}}} \mathcal{M}_{0}, \psi\right)+\int_{0}^{t} c\left(e^{(t-\tau) Q_{\mathrm{c}}} \mathcal{U}, \psi\right) \mathrm{d} \tau \\
c\left(\mathcal{S}_{\mathrm{nc}}(t), \psi\right)=c\left(e^{t Q_{\mathrm{nc}}} \mathcal{M}_{0}, \psi\right)+\int_{0}^{t} c\left(e^{(t-\tau) Q_{\mathrm{nc}}} \mathcal{U}, \psi\right) \mathrm{d} \tau
\end{gathered}
$$

where the property has been used that $c\left(\int_{0}^{t} e^{(t-\tau) Q} \mathcal{U} \mathrm{d} \tau, \psi\right)=\int_{0}^{t} c\left(e^{(t-\tau) Q} \mathcal{U}, \psi\right) \mathrm{d} \tau$. We bear on the following property of the Hausdorff metric to characterize the difference between the respective reachable sets

$$
h\left(\mathcal{S}_{\mathrm{c}}(t), \mathcal{S}_{\mathrm{nc}}(t)\right)=\max _{\psi \in \mathcal{B}_{n}(0,1)}\left|c\left(\mathcal{S}_{\mathrm{c}}(t), \psi\right)-c\left(\mathcal{S}_{\mathrm{nc}}(t), \psi\right)\right| .
$$

The difference of support functions in (37) can be written as

$$
\begin{aligned}
& c\left(\mathcal{S}_{\mathrm{c}}(t), \psi\right)-c\left(\mathcal{S}_{\mathrm{nc}}(t), \psi\right)=c\left(e^{t Q_{\mathrm{c}}} \mathcal{M}_{0}, \psi\right)-c\left(e^{t Q_{\mathrm{nc}}} \mathcal{M}_{0}, \psi\right) \\
& \quad+\int_{0}^{t}\left[c\left(e^{(t-\tau) Q_{\mathrm{c}}} \mathcal{U}, \psi\right)-c\left(e^{(t-\tau) Q_{\mathrm{nc}}} \mathcal{U}, \psi\right)\right] \mathrm{d} \tau .
\end{aligned}
$$

For the integral term in (38) we find

$$
\begin{aligned}
& \int_{0}^{t}\left[c\left(\sum_{k=0}^{\infty}(t-\tau)^{k} \frac{Q_{\mathrm{c}}^{k}}{k !} \mathcal{U}, \psi\right)-c\left(\sum_{k=0}^{\infty}(t-\tau)^{k} \frac{Q_{\mathrm{nc}}^{k}}{k !} \mathcal{U}, \psi\right)\right] \mathrm{d} \tau \\
& =\sum_{k=0}^{\infty} \int_{0}^{t}(t-\tau)^{k} \mathrm{~d} \tau\left[c\left(\frac{Q_{\mathrm{c}}^{k}}{k !} \mathcal{U}, \psi\right)-c\left(\frac{Q_{\mathrm{nc}}^{k}}{k !} \mathcal{U}, \psi\right)\right],
\end{aligned}
$$

which can be solved as

$$
c\left(Q_{\mathrm{c}}^{-1}\left(e^{t Q_{\mathrm{c}}}-I_{n}\right) \mathcal{U}, \psi\right)-c\left(Q_{\mathrm{nc}}^{-1}\left(e^{t Q_{\mathrm{nc}}}-I_{n}\right) \mathcal{U}, \psi\right)
$$

when $Q_{\mathrm{c}}$ and $Q_{\mathrm{nc}}$ are non-singular, and

$$
c\left(t \sum_{k=0}^{\infty} \frac{1}{k+1} \frac{t^{k} Q_{\mathrm{c}}^{k}}{k !} \mathcal{U}, \psi\right)-c\left(t \sum_{k=0}^{\infty} \frac{1}{k+1} \frac{t^{k} Q_{\mathrm{nc}}^{k}}{k !} \mathcal{U}, \psi\right)
$$

when $Q_{\mathrm{c}}$ and $Q_{\mathrm{nc}}$ are singular. Defining $A, B, C$ and $D$ as in Lemma 1, (34), 
and (35), the Hausdorff metric can now be bounded as follows

$$
\begin{aligned}
& h\left(\mathcal{S}_{\mathrm{c}}(t), \mathcal{S}_{\mathrm{nc}}(t)\right)=\max _{\psi \in \mathcal{B}_{n}(0,1)}\left|c\left(\mathcal{S}_{\mathrm{c}}(t), \psi\right)-c\left(\mathcal{S}_{\mathrm{nc}}(t), \psi\right)\right| \\
& =\max _{\psi \in \mathcal{B}_{n}(0,1)} \mid c\left(A^{\mathrm{T}} \mathcal{M}_{0}, \psi\right)-c\left(B^{\mathrm{T}} \mathcal{M}_{0}, \psi\right) \\
& +c\left(C^{\mathrm{T}} \mathcal{U}, \psi\right)-c\left(D^{\mathrm{T}} \mathcal{U}, \psi\right) \mid \\
& =\max _{\psi \in \mathcal{B}_{n}(0,1)} \mid c\left(\mathcal{M}_{0}, A \psi\right)-c\left(\mathcal{M}_{0}, B \psi\right) \\
& +c(\mathcal{U}, C \psi)-c(\mathcal{U}, D \psi) \mid \\
& \leq \max _{\psi \in \mathcal{B}_{n}(0,1)}\left|c\left(\mathcal{M}_{0}, A \psi\right)-c\left(\mathcal{M}_{0}, B \psi\right)\right| \\
& +|c(\mathcal{U}, C \psi)-c(\mathcal{U}, D \psi)| \\
& \stackrel{(a)}{\leq} \max _{\psi \in \mathcal{B}_{n}(0,1)} \sup _{m \in \mathcal{M}_{0}}\|m\|\|(A-B) \psi\|+\sup _{u \in \mathcal{U}}\|u\|\|(C-D) \psi\| \\
& \stackrel{(b)}{\leq} \max _{\psi \in \mathcal{B}_{n}(0,1)}\|\psi\|\left(\sup _{m \in \mathcal{M}_{0}}\|m\| \sqrt{\sum_{i, j}(A-B)_{i j}^{2}}\right. \\
& \left.+\sup _{u \in \mathcal{U}}\|u\| \sqrt{\sum_{i, j}(C-D)_{i j}^{2}}\right) \\
& =\sup _{m \in \mathcal{M}_{0}}\|m\| \sqrt{\sum(A-B)_{i j}^{2}}+\sup _{u \in \mathcal{U}}\|u\| \sqrt{\sum(C-D)_{i j}^{2}},
\end{aligned}
$$

where $(a)$ follows from the property of support functions that $\left|c\left(\mathcal{F}, \psi_{1}\right)-c\left(\mathcal{F}, \psi_{2}\right)\right| \leq$ $\sup _{f \in \mathcal{F}}\|f\|\left\|\psi_{1}-\psi_{2}\right\|$, and where $(b)$ follows from $\langle x, \psi\rangle \leq\|x\|\|\psi\|$. This concludes the proof.

The presented upper bound for the Hausdorff distance between the reachable sets of conservative and non-conservative networks provides us with a metric to compare the variation of network dynamics following from different update rules. Some special cases are listed here

(i) In the case of symmetric networks, we have $Q_{\mathrm{c}}=Q_{\mathrm{nc}}$. We find that $h\left(\mathcal{S}_{\mathrm{c}}(t), \mathcal{S}_{\mathrm{nc}}(t)\right)=0$, such that $\mathcal{S}_{\mathrm{c}}(t)=\mathcal{S}_{\mathrm{nc}}(t)$, or the reachable set is the same for conservative and non-conservative network dynamics when the system matrix is symmetric.

(ii) In the case of networks with link directions inversed, we have $Q_{\mathrm{c}}=Q_{\mathrm{nc}}^{\mathrm{T}}$. In this case, it can be demonstrated easily that $B=A^{\mathrm{T}}$ and $D=C^{\mathrm{T}}$. The Hausdorff distance can in this case be upper bounded as

$$
\begin{aligned}
h\left(\mathcal{S}_{\mathrm{c}}(t), \mathcal{S}_{\mathrm{nc}}(t)\right) \leq & \sup _{m \in \mathcal{M}_{0}}\|m\| \sqrt{\sum\left(A-A^{\mathrm{T}}\right)_{i j}^{2}} \\
& +\sup _{u \in \mathcal{U}}\|u\| \sqrt{\sum\left(C-C^{\mathrm{T}}\right)_{i j}^{2}} .
\end{aligned}
$$

This formula quantifies the increase of the Hausdorff distance as a function of the network asymmetry. 
Apart from characterizing the Hausdorff distance between reachable sets of conservative and non-conservative networks, the support function is also very powerful for other purposes. First, since the support function uniquely defines the reachable set under given constraints on the control variables, the proposed methodology can be used to analyze the minimum time to reach a given network state. The method can also be used to study the speed of expansion of the reachable set under a given update protocol. We note here that the support function allows us to verify if sets intersect by applying

$$
\mathcal{S}_{\mathrm{c}}(t) \cap \mathcal{S}_{\mathrm{nc}}(t) \neq \emptyset \Leftrightarrow c\left(\mathcal{S}_{\mathrm{c}}(t), \psi\right)+c\left(\mathcal{S}_{\mathrm{nc}}(t),-\psi\right) \geq 0, \forall \psi \in \mathbb{R}^{n} .
$$

Furthermore, we can verify if $\mathcal{S}_{\mathrm{c}}(t) \subset \mathcal{S}_{\text {nc }}(t)$, by applying

$$
\mathcal{S}_{\mathrm{c}}(t) \subset \mathcal{S}_{\mathrm{nc}}(t) \Leftrightarrow c\left(\mathcal{S}_{\mathrm{c}}(t), \psi\right) \leq c\left(\mathcal{S}_{\mathrm{nc}}(t), \psi\right), \forall \psi \in \mathbb{R}^{n} .
$$

Note that the verification of intersection and inclusion usually requires numerical analysis.

\section{Conclusion}

In this work, we proposed a framework that translates two probabilistic update protocols with constant and variable total network quantity into the expected dynamics of multi-agent networks. By including asymmetric updates and weighted links, we examined general convergence behavior and temporal dynamics in conservative and non-conservative networks. We included external control in the framework and demonstrated that both protocols express positive linear systems. The application domain of the framework is therefore catered to many practical scenarios with positive state variables. Our framework allows to study individual trajectories in terms of the dynamical and stationary properties. In particular, we stated the role of the network structure, the update rule, and the external excitation in the characteristics of the controlled output. In addition, we presented a method to analyze the set of trajectories under control constraints by examining the reachable set. Based on the support function, the method enables us to measure the difference between reachable sets of networks that operate under different protocols. Through our results, we enable both the comparison and micromanagement of network dynamics according to conservative and non-conservative updating. Future work in line with this research could transfer the proposed framework to applied problems and exploit the powerful

method of the support function to calculate the minimum time to reach desired network states.

\section{References}

[1] W. H. R. Chan, M. Wildemeersch, T. Q. S. Quek, Diffusion control in multiagent networks, in: IEEE Conference on Decision and Control (CDC), pp. 4190-4195. 
[2] A. Jadbabaie, J. Lin, A. S. Morse, Coordination of groups of mobile autonomous agents using nearest neighbor rules, IEEE Trans. Automat. Contr. 48 (2003) 988-1001.

[3] A. G. Dimakis, S. Kar, J. M. Moura, M. G. Rabbat, A. Scaglione, Gossip algorithms for distributed signal processing, Proc. of the IEEE 98 (2010) $1847-1864$.

[4] F. Pasqualetti, S. Zampieri, F. Bullo, Controllability metrics, limitations and algorithms for complex networks, IEEE Trans. Control Netw. Syst. 1 (2014) 40-52.

[5] J. Chen, A. H. Sayed, Distributed pareto optimization via diffusion strategies, IEEE J. Sel. Topics Signal Process. 7 (2013) 205-220.

[6] X. Wang, Y. Hong, Finite-time consensus for multi-agent networks with second-order agent dynamics, IFAC Proceedings Volumes 41 (2008) 1518515190 .

[7] A. V. Proskurnikov, Consensus in symmetric multi-agent networks with sector nonlinear couplings, IFAC Proceedings Volumes 44 (2011) 12371242.

[8] D. Acemoglu, A. Ozdaglar, Opinion dynamics and learning in social networks, Dynamic Games and Applications 1 (2011) 3-49.

[9] E. Mallada, R. Freeman, et al., Distributed synchronization of heterogeneous oscillators on networks with arbitrary topology, IEEE Trans. Control Netw. Syst. 3 (2016) 12-23.

[10] Ö. F. Erkan, M. Akar, Discrete-time analysis of multi-agent networks with multiple consensus equilibria, IFAC-PapersOnLine 49 (2016) 389-394.

[11] V. Blondel, J. M. Hendrickx, A. Olshevsky, J. Tsitsiklis, Convergence in multiagent coordination, consensus, and flocking, in: IEEE Conference on Decision and Control, and the European Control Conference, Seville, Spain, p. 2996.

[12] M. E. Yildiz, A. Scaglione, A. Ozdaglar, Asymmetric information diffusion via gossiping on static and dynamic networks, in: Proc. of the IEEE Conference on Decision and Control, Atlanta, GA, USA, pp. 7467-7472.

[13] G. Como, K. Savla, D. Acemoglu, M. A. Dahleh, E. Frazzoli, Robust distributed routing in dynamical flow networks, in: IEEE Conf. on Decision and Control and European Control Conference (CDC-ECC), Orlando, FL, USA, pp. 6290-6295.

[14] D. Acemoglu, G. Como, F. Fagnani, A. Ozdaglar, Opinion fluctuations and disagreement in social networks, Mathematics of Operations Research 38 (2013) 1-27. 
[15] A. Banerjee, J. Jost, On the spectrum of the normalized graph laplacian, Linear algebra and its applications 428 (2008) 3015-3022.

[16] X. Yan, S.-H. Teng, K. Lerman, R. Ghosh, Capturing the interplay of dynamics and networks through parameterizations of Laplacian operators, PeerJ Computer Science 2 (2016) e57.

[17] R. Olfati-Saber, J. A. Fax, R. M. Murray, Consensus and cooperation in networked multi-agent systems, Proc. of the IEEE 95 (2007) 215-233.

[18] D.-W. Tcha, S. R. Pliska, Optimal control of single-server queuing networks and multi-class M/G/1 queues with feedback, Operations Research 25 (1977) 248-258.

[19] R. Olfati-Saber, R. M. Murray, Consensus problems in networks of agents with switching topology and time-delays, IEEE Trans. Automat. Contr. 49 (2004) 1520-1533.

[20] D. Acemoglu, A. Nedic, A. Ozdaglar, Convergence of rule-of-thumb learning rules in social networks, in: IEEE Conference on Decision and Control, Cancun, Mexico, pp. 1714-1720.

[21] B. Barzel, A.-L. Barabási, Universality in network dynamics, Nature physics 9 (2013) 673-681.

[22] I. Mirzaev, J. Gunawardena, Laplacian dynamics on general graphs, Bulletin of mathematical biology 75 (2013) 2118-2149.

[23] M. Thompson, R. Ellis, A. Wildavsky, Cultural theory, Westview Press, 1990.

[24] A. Tversky, D. Kahneman, Availability: A heuristic for judging frequency and probability, Cognitive psychology 5 (1973) 207-232.

[25] L. Farina, S. Rinaldi, Positive linear systems: theory and applications, volume 50, John Wiley \& Sons, 2011.

[26] E. B. Lee, L. Markus, Foundations of Optimal Control Theory, Krieger, 1967.

[27] A. B. Kurzhanskiü, I. Vályi, Ellipsoidal calculus for estimation and control, Birkhäuser, 1997.

[28] R. T. Rockafellar, Convex Analysis, Princeton University Press, 1970. 